\section{Oromandibular dystonia in Yemeni patients with khat chewing: a response to botulinum toxin treatment}

\author{
Hatem S. Shehata, 1 \\ Mohamed S. El-Tamawy, ${ }^{1}$ \\ Nevin Mohieldin, 1 Mohammed Edrees, 1 \\ Saeed Bohlega2 \\ 1Department of Neurology, Cairo \\ University, Egypt; 2Department of \\ Neurosciences, King Faisal Specialist \\ Hospital and Research Centre, Riyadh, \\ Saudi Arabia
}

\section{Abstract}

Khat-(Catha edulis)related oromandibular dystonia is a difficult-to-treat subset of movement disorders that involve masticatory muscles with diverse and incapacitating manifestations. The aim of this study was to evaluate the efficacy of Botulinum toxin-type A therapy in khat chewer Yemeni patients with oromandibular dystonia. This prospective study included 18 khat-chewers Yemeni patients with refractory oromandibular dystonia, who were subjected to Botulinum toxin-A injection and followed up for 3 months thereafter. Primary efficacy outcome was the global impression scale, and secondary outcome measure was the Unified Dystonia Rating Scale. Patients showed improvement of both efficacy measures, maximum satisfactory responses were detected at the forth week after injection. No major adverse events were detected. Botulinum toxin-A is considered an effective and safe treatment option for refractory oromandibular dystonia in khat-chewers

\section{Introduction}

Oromandibular dystonia is a focal or segmental cranio-cervical dystonia, with diverse etiologies; most of which are idiopathic (primary), ${ }^{1}$ but medication-induced; ${ }^{2}$ other secondary causes like peripheral trauma and dental maneuver, 3,4 could be associated in some cases. The relationship between oromandibular dystonia and khat chewing (also known as Qat and Kat) was rarely addressed in literature. Khat is a slow-growing shrub indigenous to Yemen, Arabian Peninsula and certain parts of eastern Africa, ${ }^{5,6}$ where it has been used as a recreational drug rather than addiction. ${ }^{7}$ Its fresh leaves of Catha edulis contains psychoactive ingredients that mediate its sympath- omimetic effects known as cathinone, chemically similar to d-amphetamine and cathine, a milder form that contain D-norisoephedrine. ${ }^{8,9}$ These substances release serotonin and dopamine in central nervous system and noradrenaline from peripheral sympathetic neurons. ${ }^{10}$ On chewing khat, these constituents are released and produce excitation and increased motor activity.

The common neuropsychiatric consequences of khat chewing are memory impairment, depression and psychosis. 11 It also affects sleep, leading to rebound effects and excessive day-time sleepiness, 12 and lower seizure threshold. ${ }^{13}$ In our practice, there are mounting evidences that could suggest a causal relationship between khat and development of oromandibular dystonia (OMD).

Management of OMD is challenging. Systemic pharmacologic agents had limited efficacies; ${ }^{14}$ with no surgical alternatives apart from some trials of bilateral pallidal deep brain stimulation. ${ }^{15}$ Hence, Botulinum toxin-type A (BTX-A) is considered the treatment of choice for OMD, with robust improvement and very minimal untoward effects. ${ }^{14,16-18}$

The aim of this study is to assess the therapeutic effects of BTX-A therapy in khat chewers Yemeni patients with OMD.

\section{Materials and Methods}

\section{Study protocol}

This was a prospective, multi-center study: we took into consideration two centers in Egypt and one center in Saudi Arabia. Each of these 3 centers had a specialized movement disorder clinic. The databases in these centers were reviewed. We were able to study 18 khat chewers Yemeni patients with $\mathrm{OMD}$, who were followed up for 3 months to evaluate the therapeutic effects of botulinum toxin-A, BTX (BOTOX®, Allergan Pharmaceuticals, Irvine, CA, USA). Prior to the study commencement, all patients gave a written informed consent approved by Neurology Department Review Boards.

\section{Inclusion criteria}

The inclusion criteria were: i) chronic khat chewers with OMD who were refractory to other treatment options which were offered for at least one month; ii) symptoms that were sufficiently impairing patients' quality of life or interfering with activities of daily living; iii) patients who are not previously subjected to BTX-A injection; iv) otherwise normal neurological examination and normal brain MRI without any other apparent cause; and v) coherence of follow up visits.
Correspondence: Saeed Bohlega, Department of Neurosciences, King Faisal Specialist Hospital and Research Center, P.0. Box 3354, Riyadh 11211, Saudi Arabia.

Tel. +966.114.647.272 ext. 32827 - Fax: +966.114.424.763. E-mail: boholega@kfshrc.edu.sa

Key words: khat, oromandibular dystonia, Unified Dystonia Rating Scale, global impression scale botulinum toxin.

Contributions: the authors contributed equally.

Conflict of interests: the authors declare no potential conflict of interests.

Received for publication: 6 March 2014.

Accepted for publication: 7 April 2014.

This work is licensed under a Creative Commons Attribution NonCommercial 3.0 License (CC BYNC 3.0).

(C) Copyright H.S. Shehata et al., 2014

Licensee PAGEPress, Italy

Neurology International 2014; 6:5385

doi:10.4081/ni.2014.5385

\section{Screening phase}

Prior to the study, included patients were subjected to history review, physical and neurological examination. All of our included patients had their routine hematological and biochemical profile and MRI brain.

Nine out of the included 18 patients (50\%) had jaw opening dystonia (JOD), 7 (39\%) has jaw closure dystonia (JCD) and two patients (11\%) had mixed jaw dystonia (MJD), (a combination of jaw deviation and jaw opening). A known secondary etiology was exclusionary and in all patients khat chewing was proposed as a causative factor for their condition.

\section{Evaluation phase and follow up}

All patients were evaluated in the follow up visits at 2, 4, 8 and 12 weeks after injection. They were videotaped once before injection and 2 weeks following injection after receiving a signed release form. The uniform videotape protocol of mouth and lower face was adopted from Dystonia Study Group Videotape examination protocol. ${ }^{19}$ Jaw and lower face scoring in relation to duration and motor severity was quantified according to Unified Dystonia Rating Scale (UDRS) (jaw and tongue region). ${ }^{20}$

\section{Injection technique}

Patients' charts were used for muscle selection based on clinical assessment coupled with electromyographic (EMG) monitoring and injection was done using an Allergan ${ }^{\circledR}$ EMG needle. According to Tintner and Jankovic, 21 
submentalis, anterior belly of digastric and lateral pterygoids muscles are the potential injection targets for JOD, masseters and temporalis $(+/-)$ medial pterygoids muscles for JCD; and contralateral lateral pterygoid muscle for jaw deviation. The formulation and preparation of botulinum toxin type A (BOTOX®, Allergan Pharmaceuticals) was performed according to standard methods. ${ }^{17}$ Injected dosage regimen of BTX-A was guided by Baylor College of Medicine and Tintner and Jankovic. ${ }^{18,21}$

\section{Outcome measures}

Primary outcome measure was the global impression scale (GIS) ( $0=$ no improvement; $1=$ mild improvement; 2 = moderate improvement; 3 = marked improvement), ${ }^{22}$ while the secondary outcome measure was UDRS scoring system. ${ }^{20}$

\section{Safety measures}

Any reported adverse event during the study was recorded and graded for severity (mild, moderate, or severe). Its relationship to study treatment was (none, possible, probable, or definite). A serious adverse event was defined as one that was fatal, life-threatening, permanently disabling, or required hospitalization.

\section{Data analysis}

All statistical analysis were performed with the SPSS 15.0 software (SPSS Inc., Chicago, IL, USA). The standard descriptive statistics (e.g., mean, standard deviation) were used to summarize the data. For comparison of two variables measured in the same sample, we customarily used the t-test for dependent samples at $5 \%$ level of significance $(\alpha=0.05)$.

\section{Results}

\section{Epidemiological characteristics}

The current study included 18 patients; 12 males (67\%) and 6 females (33\%). Age range: $30-53$ years (mean, $41.89 \pm 6.69$ ). The duration of OMD ranged from 2 to 5 years (mean, $3.06 \pm 0.94$ ). Other types of dystonia were noted in 5 patients (28\%). Basic clinical characteristics are shown in Table 1.

\section{Injection techniques}

During injection session, muscle selection was based on clinical assessment and electromyography monitoring. Injected muscles are demonstrated in Table 2. The most frequently injected muscles were submentalis and anterior diagastric (11 injection for each), the least frequently injected muscles were medial pterygoids (2 injections) and lateral pterygoids (4 injections). Doses of BTX-A for each muscle are shown in Table 3.

\section{Outcome measures}

Primary efficacy variable (GIS) and secondary efficacy variable (UDRS) at 2, 4, 8, and 12 weeks interval following injection session are illustrated in Table 4.

Patients reported improvement of their symptoms (reflected on GIS) and most of our patients have noted their improvement within the first week after injection.

\section{Primary efficacy variables}

Maximum response was detected at 4 th week assessment; $56 \%(10 / 18)$ of our patients showed marked improvement (GIS: 3), 7 of them had JCD and 3 patients with JOD; whereas the other 8 patients (44\%) showed moderate improvement (GIS: 2); 6 had JOD and 2 had MJD.

\section{Secondary efficacy variables}

Patients showed response on UDRS scoring with detected maximum improvement during visit 3 (4th week) (Figure 1), and this improvement was maintained till the end of study visits (12th week).

\section{Safety measures}

There were no systemic adverse reactions detected in our patients series. Local adverse events were mild and transient; included lip numbness $(\mathrm{n}=2)$ and pain at site of injection $(n=4)$; both events disappeared within the same day of injection.

\section{Discussion and Conclusions}

Oromandibular dystonia refers to involuntary spasms of masticatory, facial and lingual muscles leading to repetitive or sustained jaw opening, closure, deviation, or any combination of these movements. Successful treatment with botulinum toxin has been demonstrated by many authorities. ${ }^{16-18,23}$ In our series we used BTX injection in chronic khat chewers Yemeni patients with refractory OMD.

Though the relationship between khat chewing and development of OMD is not clear;

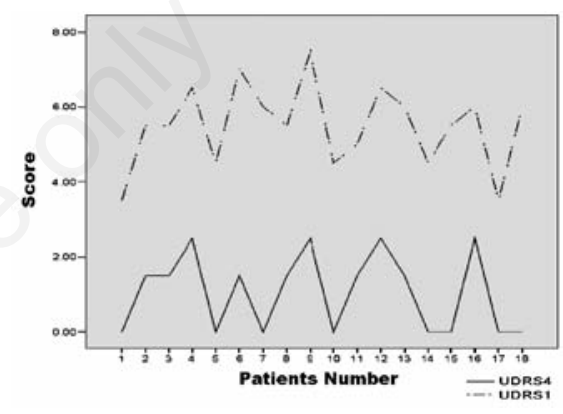

Figure 1. Unified Dystonia Rating Scale (UDRS) at $4^{\text {th }}$ week and UDRS before injection.

Table 1. Basic clinical characteristics.

\begin{tabular}{|c|c|c|c|c|c|}
\hline & $\begin{array}{l}\text { Age/ } \\
\text { Sex }\end{array}$ & $\begin{array}{l}\text { Type of } \\
\text { OMD }\end{array}$ & $\begin{array}{c}\text { Duration, } \\
\text { years }\end{array}$ & $\begin{array}{l}\text { Other types } \\
\text { of dystonia }\end{array}$ & UDRS \\
\hline 1 & $33 / \mathrm{M}$ & JCD & 2 & & 3.5 \\
\hline 2 & $45 / \mathrm{F}$ & JOD & 3 & & 5.5 \\
\hline 3 & $38 / \mathrm{M}$ & $\mathrm{JCD}$ & 4 & & 5.5 \\
\hline 4 & $41 / \mathrm{M}$ & JOD & 2 & Lower face grimacing, tongue and lip biting & 6.5 \\
\hline 5 & $35 / \mathrm{F}$ & JCD & 3 & & 4.5 \\
\hline 6 & $52 / \mathrm{F}$ & JOD & 2 & Facial grimacing and retrocollis & 7 \\
\hline 7 & $39 / \mathrm{M}$ & JOD & 4 & & 6 \\
\hline 8 & $46 / \mathrm{M}$ & JOD & 3 & & 5.5 \\
\hline 9 & $47 / \mathrm{M}$ & JOD & 4 & & 7.5 \\
\hline 10 & $30 / \mathrm{F}$ & $\mathrm{JCD}$ & 5 & & 4.5 \\
\hline 11 & $40 / \mathrm{F}$ & JOD & 3 & & 5 \\
\hline 12 & $33 / \mathrm{M}$ & MJD & 3 & JDD, JOD and retrocollis & 6.5 \\
\hline 13 & $48 / \mathrm{M}$ & MJD & 2 & JDD, JOD, lower facial grimacing and tongue protrusion & n 6 \\
\hline 14 & $43 / \mathrm{M}$ & JOD & 4 & & 4.5 \\
\hline 15 & $49 / \mathrm{F}$ & JCD & 2 & & 5.5 \\
\hline 16 & $53 / \mathrm{M}$ & JOD & 3 & Facial grimacing & 6 \\
\hline 17 & $44 / \mathrm{M}$ & JCD & 4 & & 3.5 \\
\hline 18 & $38 / \mathrm{M}$ & JCD & 2 & & 6 \\
\hline
\end{tabular}


yet, dystonia and dyskinesias can be induced by khat as amphetamine-containing compounds are known inducer for movement disorders. ${ }^{24}$ There are few reports that addressed this relationship. Harms et al. ${ }^{25}$ proposed this relationship, and to our knowledge, there were no other studies that could recognize it as a causal factor. In 1994, Thiel and Dressler reported cases of previously healthy patients, who developed persistent dyskinetic syndromes (spasmodic torticollis and cranial dys- tonia) following the intake of appetite suppressant that contains norpseudoephedrine, which is pharmacologically related to amphetamine. ${ }^{24}$ Beside the chemical related effects of khat, a mechanical effect could be proposed as khat chewing or (better replaced by the Arabic word Takhzeen, which implicates placing the green-leaved plant into the mucobuccal fold and chewing it for several hours $)^{26}$ could be considered as task-specific dystonia similar to writers' cramps. In our series, jaw opening dys-
Table 2. Muscle selection for injection.

\begin{tabular}{ll}
\hline 1 & Muscle selection for injection \\
2 & Sasseters and temporalis \\
\hline 3 & Masseters, temporalis and medial pterygoids \\
\hline 4 & Submentalis and anterior digastrics \\
\hline 5 & Masseters, temporalis \\
\hline 7 & Submentalis, anterior digastric and lateral pterygoids \\
\hline 8 & Submentalis and anterior digastrics \\
\hline 9 & Submentalis and anterior digastrics \\
\hline 10 & Submentalis, anterior digastric and lateral pterygoids \\
\hline 11 & Masseters and temporalis \\
\hline 13 & Submentalis and anterior digastrics \\
\hline 14 & Submentalis, anterior digastric and lateral pterygoids \\
\hline 15 & Submentalis, anterior digastric and lateral pterygoids \\
\hline 16 & Submentalis and anterior digastrics \\
\hline 17 & Masseters and temporalis \\
\hline 18 & Submentalis and anterior digastrics \\
\hline
\end{tabular}

tonia is the most encountered type in khat chewer (50\%); whereas, 39\% has jaw closure dystonia (JCD). On the contrary, jaw closing dystonia was more frequently recorded in Baylor College of Medicine (BCM) and Tintner, ${ }^{18}$ and Jankovic, ${ }^{21}$ being $53 \%$ and $53 \%$ respectively; this might point to the type of khat chewing associated dystonia.

During injection, we adopted previously reported techniques, ${ }^{21}$ and basically our injection paradigm was manipulated according to clinical assessment and EMG guidance. Gelb et $a l .{ }^{27}$ reported that it is not always possible to use electromyography in all involved muscles in OMD due to technical difficulties and in some cases, the pattern of muscle involvement may change over time. However, as documented previously, ${ }^{28}$ better efficiency and longer response duration are noted when injections are guided by electromyography.

The mean doses of BTX-A in submentalis were $29 \pm 2.24$ Mouse Units (U) and $61.25 \pm 11.57 \mathrm{U}$ in masseters (per side). These

Table 3. Dose of botulinum toxin injection for each muscles.

\begin{tabular}{lcc} 
Injected muscle & \multicolumn{2}{c}{ Dose of botulinum toxin } \\
& Range, units & Mean (SD) \\
Submentalis & $25-30$ & $29(2.24)$ \\
Anterior digastrics & $20-30$ & $25(3.53)$ \\
\hline Masseter & $40-75$ & $61.25(11.57)$ \\
Temporalis & $40-60$ & $46.88(8.84)$ \\
\hline Medial pterygoids & $20-30$ & $25(7.07)$ \\
Lateral pterygoids & $20-35$ & $27.5(10.61$ \\
\hline
\end{tabular}

Table 4. Primary (GIS) and secondary (UDRS) efficacy variables at 2, 4, 8, and 12 weeks after injection.

\begin{tabular}{|c|c|c|c|c|c|c|c|c|}
\hline & \multicolumn{2}{|c|}{2 weeks assessment } & \multicolumn{2}{|c|}{4 weeks assessment } & \multicolumn{2}{|c|}{8 weeks assessment } & \multicolumn{2}{|c|}{12 weeks assessment } \\
\hline & GIS & UDRS(OMD) & GIS & UDRS (OMD) & GIS & UDRS (OMD) & & UDRS (OMD) \\
\hline 1 & 1 & 1.5 & 3 & 0.0 & 3 & 0.0 & 3 & 0.0 \\
\hline 2 & 1 & 2.5 & 2 & 1.5 & 2 & 1.5 & 2 & 1.5 \\
\hline 3 & 2 & 2.5 & 3 & 1.5 & 3 & 1.5 & 3 & 1.5 \\
\hline 4 & 1 & 3.0 & 2 & 2.5 & 2 & 2.5 & 2 & 2.5 \\
\hline 5 & 3 & 1.5 & 3 & 0.0 & 3 & 0.0 & 3 & 0.0 \\
\hline 6 & 2 & 3.0 & 3 & 1.5 & 3 & 1.5 & 3 & 1.5 \\
\hline 7 & 2 & 2.5 & 3 & 0.0 & 3 & 0.0 & 3 & 0.0 \\
\hline 8 & 1 & 3.0 & 2 & 1.5 & 2 & 1.5 & 2 & 1.5 \\
\hline 9 & 1 & 3.5 & 2 & 2.5 & 2 & 2.5 & 2 & 2.5 \\
\hline 10 & 2 & 1.5 & 3 & 0.0 & 3 & 0.0 & 3 & 0.0 \\
\hline 11 & 1 & 2 & 2 & 1.5 & 2 & 1.5 & 2 & 1.5 \\
\hline 12 & 2 & 2.5 & 2 & 2.5 & 2 & 2.5 & 2 & 2.5 \\
\hline 13 & 2 & 2.0 & 2 & 1.5 & 2 & 1.5 & 2 & 1.5 \\
\hline 14 & 3 & 1.5 & 3 & 0.0 & 3 & 0.0 & 3 & 0.0 \\
\hline 15 & 2 & 2.0 & 3 & 0.0 & 3 & 0.0 & 3 & 0.0 \\
\hline 16 & 2 & 2.5 & 2 & 2.5 & 2 & 2.5 & 2 & 2.5 \\
\hline 17 & 2 & 2.0 & 3 & 0.0 & 3 & 0.0 & 3 & 0.0 \\
\hline 18 & 3 & 1.5 & 3 & 0.0 & 3 & 0.0 & 3 & 0.0 \\
\hline
\end{tabular}

GIS, global impression scale; UDRS, unified dystonia rating scale; OMD, oromandibular dystonia. 
2009;105:18-21.

doses are nearly similar to those used in BCM study. ${ }^{18}$ Regarding other injected muscles, we used higher mean doses than that were injected by Tintner and Jankovic. ${ }^{21}$ Noteworthy, there are no clear guidelines for precise dosages for each injected muscle, and doses depend upon different methods of patient and muscle selection and different injection techniques. In our study, higher mean doses were attributed to the markedly increased muscle activities that were detected during EMG assessment.

The beneficial effects of BTX-A in khat chewers with OMD were obvious in our work. The overall effect of BTX-A injection was satisfactory for all included patients, with no response failure. Complete objective improvement (UDRS score 0) was detected in 8/18 (44\%) of our patients at 4th week following injection, and it was maintained till 12 th week post-injection. These results were previously addressed by numerous studies, $17,18,23$ with a response rate reached up to $90-95 \%$.

In the current study, we could not detect any major adverse events; this might be explained by EMG-guidance methodology and injecting the most anterior portion of submental complex. In conclusion, BTX-A is considered a satisfactory treatment option for khat chewers with OMD with high safety and tolerability.

\section{References}

1. Burke RE, Fahn S, Jankovic J, et al. Tardive dystonia: late-onset and persistent dystonia caused by antipsychotic drugs. Neurology 1982;32:1335-46.

2. Jankovic J. Tardive syndromes and other drug-induced movement disorders. Clin Neuropharmacol 1995;18:197-214.

3. Sankhla C, Lai EC, Jankovic J. Peripherally induced oromandibular dystonia. J Neurol Neurosurg Psychiatry 1998;65:722-8.

4. Thorburn DN, Lee KH. Oromandibular dystonia following dental treatment: case reports and discussion. $\mathrm{N} Z$ Dent $\mathrm{J}$
5. Saha S, Dollery C. Severe ischaemic cardiomyopathy associated with khat chewing. J R Soc Med 2006;99:316-8.

6. Yarom N, Epstein J, Levi H, et al. Oral manifestations of habitual khat chewing: a case-control study. Oral Surg Oral Med Oral Pathol Endod 2010;109:e60-8.

7. Manghi RA, Broers B, Khan R, et al. Khat use: lifestyle or addiction. J Psychoactive Drugs 2009;41:1-10.

8. Cox G. Adverse effects of khat: a review. Adv Psychiatr Treat 2003;9:456-63.

9. Balint EE, Falkay G, Balint GA. Khat - a controversial plant. Wien Klin Wochenschr 2009;121:604-14.

10. Kalix P. A comparison of the catecholamine releasing effect of the khat alkaloids (-)-cathinone and (+)-norpseudoephidrine. Drug Alcohol Depend 1983; 11:395-401.

11. Houghton P. Khat - a growing concern in the UK. Pharmaceutic J 2004;272:163-5

12. Al-Mugahed L. Khat chewing in Yemen: turning over a new leaf. Bull World Health Organ 2008;86:741-2.

13. Oyungu E, Kioy PG, Patel NB. Proconvulsant effect of khat (Catha edulis) in Sprague dawley rat. J Ethnopharmacol 2009;121:476-8.

14. Jankovic J. Medical therapy and botulinum toxin in dystonia. Adv Neurol 1998;78:16983.

15. Markaki E, Kefalopoulou Z, Georgiopoulos M, et al. Meige's syndrome: a cranial dystonia treated with bilateral pallidal deep brain stimulation. Clin Neurol Neurosurg 2010;112:344-6.

16. Schwartz M, Freund B. Treatment of temporomandibular disorders with botulinum toxin. Clin J Pain 2002;18:S198-203.

17. Jankovic J, Orman J. Botulinum A toxin for cranial-cervical dystonia: a double-blind, placebo-controlled study. Neurology 1987; 37:616-23.

18. Tan EK, Jankovic J. Botulinum toxin A in patients with oromandibular dystonia: long-term follow-up. Neurology 1999;53:
2102-7.

19. Burke RE, Fahn S, Marsden CD, et al. Validity and reliability of a rating scale for the primary torsion dystonias. Neurology 1985;35:73-7.

20. Comella CL, Leurgans S, Wuu J, et al. Rating scales for dystonia: a multicenter assessment. Mov Disord 2003;18:303-12.

21. Tintner R, Jankovic J. Botulinum toxin type $\mathrm{A}$ in the management of oromandibular dystonia and bruxism. In: Brin MF, Hallett M, Jankovic J, eds. Scientific and therapeutic aspects of botulinum toxin. Philadelphia: Lippincott Williams \& Wilkins; 2002.

22. Papapetropoulos S, Singer C. Eating dysfunction associated with oromandibular dystonia: clinical characteristics and treatment considerations. Head Face Med 2006;2:47.

23. Gadhia K, Walmsley D. The therapeutic use of botulinum toxin in cervical and maxillofacial conditions. Evid Based Dent 2009; 10:53.

24. Thiel A, Dressler D. Dyskinesias possibly induced by norpseudoephedrine. J Neurol 1994;241:167-9.

25. Harms L, Spokert F, Alwarith H, et al. Oromandibular dyskinesia and dystonia with khat chewers. Poster Presentation (352). Drug-induced Movement Disorders Session. The movement disorder society's 11th International congress of parkinson's disease and movement disorders. June 37, 2007. Istanbul, Turkey.

26. Aiman A. Comment on oral white lesions associated with chewing khat. Tob Induc Dis 2005;3:5-6.

27. Gelb DJ, Yoshimura DM, Olney RK, et al. Change in pattern of muscle activity following botulinum toxin injections for torticollis. Ann Neurol 1991;29:370-6.

28. Møller E, Bakke M, Dalager T, Werdelin LM. Oromandibular dystonia involving the lateral pterygoid muscles: four cases with different complexity. Mov Disord 2007;22: 785-90. 